\title{
Estudo de Schinus terebenthifolius na recuperação de áreas ciliares sob tratamentos convencional, orgânico e biodinâmico
}

\author{
Ciro Guilherme Gentil Croce ${ }^{1}$ \\ Maristela Simões Carmo ${ }^{1}$ \\ Francisco Luiz Araújo Câmara ${ }^{1}$ \\ Iraê Amaral Guerrini ${ }^{1}$ \\ ${ }^{1}$ Universidade Estadual Paulista - UNESP \\ Rua José Barbosa de Barros, 1780 Fazenda Lageado, Botucatu - SP, Brasil \\ \{cirogc, Stella, chicocamara, iguerrini\}@fca.unesp.br
}

\begin{abstract}
Native species have been largely studied due to their potential for reforesting degraded areas. In this way, the aim of the present study was to evaluate the initial development of one pioneer native tree specie, Schinus terebinthifolius (Aroeira-Pimenteira), in organic, conventional (NPK) and biodynamic treatments. For 6 months 43 seedlings were cultivated in each one of the tested substrates. It were measured the plant height, colon diameter, wet and dry weight, and chemical analysis of aerial and root parts of the plants were carried as well. For S. terebinthifolius the soil treatments almost did not affect of the tested characteristics, growth was still better in conventional than in organic soil, but it may be an effect of the chemical action of this treatment that has a direct effect on the plant and may bring serious soil conservation problems. The study satisfies the proposal of searching for sustained systems for the development of seedlings and recovery of degraded areas.
\end{abstract}

Keywords: biodynamic agriculture, organic agriculture, pioneer, Schinus terebinthifolius, initial growth. 


\section{INTRODUÇÃO}

As mudas mais usadas em programas de reflorestamento são as chamadas pioneiras. De acordo com Budowski(1965), as plantas pioneiras são as primeiras a colonizarem um novo ambiente, seguidas das secundárias iniciais, secundárias tardias e, finalmente, as de clímax. As plantas pioneiras são geralmente espécies que ocupam inicialmente o solo, no processo sucessional de recuperação da floresta após qualquer distúrbio. Possuem características próprias de sementes de rápida germinação e sensíveis a luz, crescimento rápido e período curto de vida.

A adubação, no desenvolvimento inicial, é uma variável que contribui significativamente para o desenvolvimento das mudas. Mas esse processo é o mais delicado em termos de melhoramento dos solos cultivados, porque nem sempre se tem, na prática, uma noção perfeita do que seja adubar com respeito às exigências do solo e da planta, isto é, a qualidade e quantidade dos adubos a se empregar. Além disso, deve-se escolher o tipo de adubo a ser aplicado, visto que existem vários disponíveis, dentre eles os minerais ou químicos, os orgânicos e, mais recentemente os biodinâmicos.

O adubo natural, termo utilizado para os adubos não minerais, na história da agricultura, é o material fertilizante mais tradicional nas áreas de cultivo, especialmente sob a forma de dejetos animais. O curtimento natural, processo utilizado na reunião de resíduos orgânicos da agricultura para posterior uso como adubo, pode ser ainda aperfeiçoado com a combinação de diversos compostos, processo esse chamado compostagem. A Agricultura Biodinâmica pretende desenvolver uma paisagem cultivada sadia, próspera e de produtividade permanente, na qual a qualidade dos alimentos seja aprimorada a partir dos cuidados com o solo. Traz uma visão abrangente de um sistema agrícola integrado, o organismo agrícola, inserido harmoniosamente na paisagem local, considerando-se os seus princípios ecológicos, sociais, técnicos, culturais, econômicos e fenomenológicos.

Nesse contexto, o presente estudo tem como objetivo acompanhar e avaliar o desenvolvimento inicial e a absorção de nutrientes de uma espécie arbórea nativa pioneira, Schinus terebinthifolius (Aroeira-Pimenteira), nos sistemas orgânico, convencional (NPK) e biodinâmico. O desenvolvimento foi acompanhado ao longo de seis meses por medições de altura, espessura de colo, peso úmido e seco e, por fim, foram feitas análises químicas das partes aéreas e radicular das mudas.

\section{MATERIAL E MÉTODOS}

\subsection{Delineamento Geral}

A estratégia básica deste trabalho consistiu em avaliar o desenvolvimento da espécie Schinus terebinthifolius Raddi em substrato com adubações convencional, orgânica e biodinâmica. Cada um dos três tratamentos teve 43 repetições, resultando em 129 mudas. O experimento foi realizado no viveiro no Departamento de Recursos Naturais - Ciências Florestais, UNESP, município de Botucatu, SP (latitude $22^{\circ} 42^{\prime} 30^{\prime}$ S e longitude $47^{\circ} 38^{\prime} 20^{\prime \prime} \mathrm{W}$ ).

A Schinus terebinthifolius Raddi (Aroeira-Pimenteira) pertence a família Anacardiaceae. São plantas arbóreas, pioneiras e nativas do Brasil que ocorrem geralmente em florestas estacionais semideciduais.

A espécie S. terebinthifolius é conhecida popularmente como Aroeira-Mansa, AroeiraPrecoce, Aroeira-Pimenteira, Aroeira-do-Campo, Balsamo, Cambuí ou outros. Atinge de 5 a $10 \mathrm{~m}$ de altura e seu tronco tem de 30 a $60 \mathrm{~cm}$ de diâmetro. Possui folhas compostas imparipinadas de 10 a $15 \mathrm{~cm}$ de comprimento. Seus frutos são muito procurados por pássaros dispersores de suas sementes. É uma planta indispensável nos reflorestamentos heterogêneos destinados à recomposição de áreas degradadas de preservação permanente (LORENZI, 2000). 


\subsection{Preparação dos Substratos}

\subsubsection{Sistema convencional (NPK)}

No substrato convencional foi usado o fertilizante NPK de fórmula 4.14.8, da marca Fertiap, comercializado em lojas agropecuárias. Foi utilizado também no tratamento convencional (químico), o calcário dolomítico em pó, da marca Minercal Agrícola.

\subsubsection{Composto Orgânico}

O composto orgânico e o biodinâmico foram elaborados com os procedimentos comuns de compostagem. Foram usados na compostagem os resíduos vegetais da empresa Anidro do Brasil que produz extratos a partir de plantas medicinais no município de Botucatu, São Paulo. A medida que chegavam as plantas no local escolhido para a compostagem, os montes foram sendo preparados com porções iguais para as duas pilhas de compostos, a orgânica e a biodinâmica. O esterco foi introduzido duas vezes durante a montagem das pilhas.

\subsubsection{Composto Biodinâmico}

Uma das pilhas de compostagem (produzida conforme Quadro 1) foi suplementada com os "preparados biodinâmicos" de 502 a 507, adquiridos na Associação Brasileira de Agricultura Biodinâmica (ABD). Esses preparados foram utilizados na dosagem de $2 \mathrm{~g} / 3,5 \mathrm{t}$ (preparado 502 a 506) e 1 a $2 \mathrm{~mL} / 3,5 \mathrm{t}$ (preparado 507). Esses preparados foram colocados conforme sistema descrito por Wistinghausen (2000), a 2 metros de distância entre si. Os preparados usados tinham as seguintes especificações:

Preparado 502 - Milfolhas (Achilea millefolium): usam-se as flores da milfolhas; trabalha os processos do potássio através do enxofre.

Preparado 503 - Camomila (Matricaria chamomilla): usam-se as flores da camomila; ativa os processos do cálcio juntamente com o enxofre.

Preparado 504 - Urtiga (Urtica dioica): usam-se folhas e caule da urtiga; ativa os processos de ferro e evita a perda de nitrogênio.

Preparado 505 - Casca de carvalho (Quercus robur): usa-se a casca do carvalho; auxilia em processos de doenças criptogâmicas, principalmente através do cálcio.

Preparado 506 - Dente-de-leão (Taraxacum officinale): usam-se as flores amarelas do dentede-leão; favorece os processos do enxofre e potássio.

Preparado 507 - Valeriana (Valeriana officinalis): este preparado regula as funções de calor pelo fósforo; faz-se a dinamização de 1 a $2 \mathrm{~mL}$ do extrato das flores da valeriana diluindo em 10 litros de água morna por 15 a 20 minutos (vide dinamização). Deixar descansar um pouco (cerca de 2 minutos) e então pulverizar sobre a pilha, formando uma capa de proteção (HERMÍNIO, 2002).

\subsubsection{Mistura dos adubos com terra}

Os substratos usados neste estudo foram elaborados com os parâmetros utilizados pelo viveiro das Ciências Florestais da UNESP de Botucatu, colocados em embalagem plástica de polietileno ( $20 \mathrm{~cm}$ de diâmetro x $45 \mathrm{~cm}$ de altura) que foram completadas com terra, de modo que todos os tratamentos tiveram o mesmo volume. Não foi necessário corrigir a quantidade de terra entre os tratamentos porque o presente estudo visou avaliar os substratos nas concentrações em que são empregados corriqueiramente em viveiro e no campo para formação de mudas e reflorestamentos de espécies nativas. Dessa forma, as embalagens de cada tratamento foram montadas nas seguintes proporções:

a) Convencional: $8,0 \mathrm{Kg}$ de terra $+0,075 \mathrm{Kg}$ de NPK (4.14.8) e 0,025 Kg de Calcário Dolomítico

b) Orgânico: $4,5 \mathrm{Kg}$ de terra $+2,5 \mathrm{Kg}$ de composto orgânico

c) Biodinâmico: $4,5 \mathrm{Kg}$ de terra $+2,5 \mathrm{Kg}$ de composto biodinâmico 


\subsection{Análises Estatísticas}

Foram realizadas análises de regressão, mês a mês, conforme crescimento em altura e diâmetro de colo.

Os valores de altura, diâmetro de colo, peso úmido e peso seco observados ao final de 180 dias, foram submetidos a análise de variância e as médias de tratamentos comparadas pelo teste de Tukey, considerando o nível de $5 \%$ de probabilidade.

Dadas as diferenças estatísticas encontradas para a absorção de nutrientes (por quilo) pelas plantas, usou-se o teste de qui-quadrado $\left(\chi^{2}\right)$ para analisar se as ocorrências de pico de concentração foram semelhantes entre os tratamentos (foram descartados os dados sem diferenças estatísticas). Uma subdivisão do teste de qui-quadrado corrigido por Yates foi aplicada para identificação dos tratamentos com pico de concentração diferentes.

\section{RESULTADOS}

Comparando-se a altura final das mudas de S. terebinthifolius (Aroeira), observou-se que as do tratamento convencional (NPK) ficaram mais altas do que as submetidas aos tratamentos orgânico e biodinâmico que, por sua vez, foram iguais entre si (ANOVA, p<0,05; Tabela 1). Comparando-se os diâmetros de colo, não foi constatada diferença entre as mudas dos tratamentos biodinâmico e convencional, e ambos tiveram valores superiores aos do tratamento orgânico (ANOVA, $\mathrm{p}<0,05$; Tabela 1).

Tabela 1. Altura e diâmetro do colo mensais de mudas de S. terebinthifolius (Aroeira) plantadas em substrato orgânico, convencional (NPK) e biodinâmico.

\begin{tabular}{llllllll}
\hline \multirow{2}{*}{ Variável } & \multirow{2}{*}{ Substrato } & \multicolumn{2}{l}{ Meses } & & & \\
& & Mai & Jun & Jul & Ago & Set & Out \\
\hline \multirow{3}{*}{ Altura (cm) } & Orgânico & 27,2 & 30,3 & 45,6 & 72,51 & 109,16 & $132,45 \mathrm{~b}$ \\
& NPK & 27,2 & 40,1 & 62,9 & 90,84 & 129,04 & $142,39 \mathrm{a}$ \\
& Biodinâmico & 25,8 & 33,3 & 59,9 & 87,22 & 108,15 & $120,53 \mathrm{~b}$ \\
\hdashline & Orgânico & 3,1 & 4,52 & 7,01 & 9,87 & 11,9 & $13,41 \mathrm{~b}$ \\
\multirow{2}{*}{ Diâmetro (cm) } & NPK & 3,3 & 5,5 & 8,67 & 11,29 & 13 & $14,31 \mathrm{a}$ \\
& Biodinâmico & 3,36 & 5,34 & 8,5 & 11,25 & 12,98 & $14,04 \mathrm{a}$ \\
\hline
\end{tabular}

Como mostrado na Tabela 2, em relação ao peso seco e úmido das folhas e raízes da Aroeira, bem como do peso seco total, não foram constatadas diferenças significativas entre os tratamentos (ANOVA, $\mathrm{p}>0,05$ ). Já o peso úmido e seco dos ramos e o peso total úmido foram maiores para as mudas do tratamento convencional em relação ao orgânico, e nenhum deles diferiu do tratamento biodinâmico (ANOVA, $\mathrm{p}<0,05$ ).

Tabela 2. Média do peso úmido e seco ( $\mathrm{g}$ ) de folhas, ramos e raízes de $S$. terebinthifolius (Aroeira) desenvolvida nos substratos orgânico, convencional (NPK) e biodinâmico.

\begin{tabular}{llllll}
\hline \multirow{2}{*}{ Variável } & \multirow{2}{*}{ Substrato } & \multicolumn{4}{l}{ Partes da Planta } \\
\cline { 3 - 6 } & & Folhas & Ramos & Raízes & Total \\
\hline \multirow{2}{*}{ Peso Úmido } & Orgânico & 78,33 & $78,85 \mathrm{~b}$ & 44,9 & $202,08 \mathrm{~b}$ \\
& Convencional & 82,31 & $114,66 \mathrm{a}$ & 61,18 & $258,15 \mathrm{a}$ \\
& Biodinâmico & 80,32 & $94,29 \mathrm{ab}$ & 53,55 & $228,16 \mathrm{ab}$ \\
\multirow{2}{*}{ Peso Seco } & Orgânico & 24,00 & $25,77 \mathrm{~b}$ & 14,96 & 64,73 \\
& Convencional & 24,62 & $37,13 \mathrm{a}$ & 18,59 & 80,34 \\
& Biodinâmico & 27,39 & $36,57 \mathrm{ab}$ & 19,66 & 83,62 \\
\hline
\end{tabular}

Para cada variável, médias seguidas de letras diferentes na coluna diferem entre si ao nível de $5 \%$ de probabilidade. 
Tabela 3. Freqüência com que o pico de concentração de nutrientes na Aroeira ocorreu em cada tratamento.

\begin{tabular}{lllll}
\cline { 2 - 4 } $\begin{array}{l}\text { Letras diferentes } \\
\text { diferenças }\end{array}$ & Planta & \multicolumn{2}{l}{ Tratamento } & \\
\cline { 2 - 4 } \begin{tabular}{l} 
os tratamentos \\
\cline { 2 - 4 } representam \\
estatísticas entre
\end{tabular} & Aroeira & $15 \mathrm{~b}$ & $29 \mathrm{a}$ & $9 \mathrm{~b}$ \\
\cline { 2 - 4 }
\end{tabular}

\section{CONCLUSÃO}

Em termos de crescimento e das características de cada substrato, a espécie S. terebinthifolius apresenta respostas diferentes em relação aos substratos orgânico, convencional (NPK) e biodinâmico. De modo geral para $S$. terebinthifolius, embora a diferença entre os tratamentos não tenha sido evidente para as características avaliadas, há um melhor desempenho no substrato com NPK do que no orgânico, e isso pode estar relacionado à ação dos elementos minerais que atuam diretamente na planta, mas pode trazer grandes desvantagens em termos de conservação do solo. S. terebinthifolius teve também um bom desenvolvimento em substrato biodinâmico. Neste estudo, pode-se considerar que as mudas de Aroeira tiveram desempenho semelhante em substrato biodinâmico e convencional, pois uma única exceção foi encontrada para altura que foi maior no tratamento convencional. Por outro lado, observou-se que as mudas desenvolvidas em substrato orgânico tiveram para altura, diâmetro e ganho total de biomassa úmida um desempenho inferior ao tratamento convencional (altura $=18 \%$ e biomassa úmida total $=28 \%$ ). Esse resultado pode ser explicado pelas características da Aroeira, que é uma planta menos exigente e portanto possui um bom desempenho até mesmo em solos mais pobres (LORENZI, 2000). Assim, pode ser que as condições favoráveis criadas pela disponibilidade de matéria orgânica no tratamento orgânico não tenham sido tão cruciais. Seu maior desempenho se deve principalmente à disponibilização de nutrientes mineralizados e prontos para absorção proporcionados pelo NPK. A análise química foliar, dos ramos e raízes confirma o processo evolutivo para a planta. Também para Aroeira, observou-se que os níveis de concentração da maior parte dos nutrientes nas partes das plantas foi mais elevado no tratamento convencional do que no orgânico e no biodinâmico.

\section{REFERÊNCIAS BIBLIOGRÁFICAS}

AGUIAR, I.B.; VALERI, S.V.; BANZATTO, D.A.; CORRADINI, L.; ALVARENGA, S.F. Seleção de componentes de substrato para produção de mudas de eucalipto em tubetes. IPEF, Piracicaba, v.41/42, p.36-43, 1989.

BUDOWSKI, G. Distribution of Tropical American rain Forest species in the light of successional processes. Turrialba, v.15, p.40-42, 1965.

CHABOUSSOU, F. Plantas doentes pelo uso de agrotóxicos. A teoria da trofobiose. Porto Alegre: Editora LPM, 1980. 253p.

EMBRAPA. Pesquisa desenvolve tecnologias para a maximização da qualidade e produtividade do Eucalipto. Embrapa - novidades - folhaflorestas, 2002. Disponível em: $<$ http://www.cnpf.embrapa.br/novidades/folhaflorestas/15/pesquisa $>$ Acesso em 7 nov. 2004 GOH, K.M.; HAYNES, R.J. Evaluation of potting media for commercial nursery production of container-grown plants. I. Physical and chemical characteristics of soil and soil-less media and their constituents. New Zeland Journal of Agriculture, v.20, p.363-370, 1997. GONÇALVES, J.L.M; POGGIANI, F. Substratos para produção de mudas florestais. Anais do Congresso Latino Americano de Ciência do Solo, 13, 1996, Águas de Lindóia, SP. 1996. 
HAUG, R.T. The practical handbook of compost engineering. Florida: Boca Raton, 1993. $717 \mathrm{p}$.

HERMÍNIO, D.B.C. Revista Agricultura Biodinâmica (ABD) v.19, n.86, p.12-14, 2002. KIEHL, E.J. Manual de Compostagem: maturação e qualidade do composto. 3.ed. Piracicaba: 2000. 171p.

LORENZI, H. Árvores Brasileiras: manual de identificação e cultivo de plantas arbóreas do Brasil. 3.ed. Nova Odessa: Instituto Plantarum, 2000. 373p.

PASCHOAL, A. D. Produção orgânica de alimentos. Piracicaba: Esalq, 1994. 191 p. PEREIRA NETO, J.T. Manual de compostagem processo de baixo custo. Belo Horizonte UNICEF, 1996. 56p.

PIRES DE MORAES NETO, S.; GONÇALVES, J.L.M..; TAKAKI, M. Produção de mudas de seis espécies arbóreas, que ocorrem nos domínios da Floresta Atlântica, com diferentes substratos de cultivo e níveis de luminosidade. Revista Árvore 2001. V.25, n.3-4, p.277-287. RODELLA, A.A.; ALCARDE, J.C. Requisitos de qualidade física e química de fertilizantes naturais. In: GONÇALVES, J.L.M.; BENEDETTI, V. (Eds.) Nutrição e Fertilização Florestal. Piracicaba: IPEF, 2000. p. 59-78.

SOCIEDADE ANTROPOSÓFICA NO BRASIL. Disponível em:

$<$ http://www.sab.org.br/agric-biod/solovivo.htm>. Acesso em 29.nov.2004.

STEINER, R. Fundamentos da agricultura biodinâmica: vida nova para a terra. São Paulo: Antroposófica, 2000. 235p.

WISTINGHAUSEN, C. Manual para uso dos preparados biodinâmicos. São Paulo: Antroposófica, 2000. 77p. 